\title{
Naphthoquinone Derivatives as Scaffold to Develop New Drugs for Tuberculosis Treatment
}

OPEN ACCESS

Edited by:

Fernando Rogerio Pavan,

Universidade Estadual Paulista "Júlio de Mesquita Filho" (UNESP),

Brazil

Reviewed by:

Katiany Rizzieri Caleffi Ferracioli, Universidade Estadual de Maringá,

Brazil

Sandeep Sharma,

University of Pennsylvania,

United States

Banasri Hazra,

Jadavpur University, India

*Correspondence:

Daniela F. Ramos

daniferamos@gmail.com

Specialty section: This article was submitted to Antimicrobials, Resistance

and Chemotherapy,

a section of the journal

Frontiers in Microbiology

Received: 12 January 2018

Accepted: 22 March 2018

Published: 09 April 2018

Citation:

Halicki $P C B$, Ferreira $L A$

De Moura KCG, Carneiro PF, Del Rio KP, Carvalho TSC,

Pinto MCFR, da Silva PEA and Ramos DF (2018) Naphthoquinone Derivatives as Scaffold to Develop

New Drugs for Tuberculosis

Treatment. Front. Microbiol. 9:673. doi: 10.3389/fmicb.2018.00673

\author{
Priscila C. B. Halicki', Laís A. Ferreira', Kelly C. G. De Moura², Paula F. Carneiro², \\ Karina P. Del Rio ${ }^{2}$, Tatiane dos S. C. Carvalho², Maria do C. F. R. Pinto', \\ Pedro E. A. da Silva ${ }^{1}$ and Daniela F. Ramos ${ }^{1 *}$
}

${ }^{1}$ Núcleo de Pesquisa em Microbiologia Médica, Faculdade de Medicina, Universidade Federal do Rio Grande, Rio Grande, Brazil, ${ }^{2}$ Núcleo de Pesquisas em Produtos Naturais, Centro de Ciências da Saúde, Universidade Federal do Rio de Janeiro, Rio de Janeiro, Brazil

Despite being a curable disease, tuberculosis (TB) remains a public health problem worldwide mainly due to lengthy treatment, as well as its toxic effects, TB/HIV coinfection and the emergence of resistant Mycobacterium tuberculosis strains. These barriers reinforcing the need for development of new antimicrobial agents, that ideally should reduce the time of treatment and be active against susceptible and resistant strains. Quinones are compounds found in natural sources and among them, the naphthoquinones show antifungal, antiparasitic, and antimycobacterial activity. Thus, we evaluated the potential antimycobacterial activity of six 1,4-naphthoquinones derivatives. We determined the minimum inhibitory concentration (MIC) of the compounds against three $M$. tuberculosis strains: a pan-susceptible H37Rv (ATCC 27294); one mono-resistant to isoniazid (ATCC 35822); and one mono-resistant to rifampicin (ATCC 35838); the cytotoxicity in the J774A.1 (ATCC TIB-67) macrophage lineage; performed in silico analysis about absorption, distribution, metabolism, and excretion (ADME) and docking sites. All evaluated naphthoquinones were active against the three strains with MIC between 206.6 and $12.5 \mu \mathrm{M}$, and the compounds with lower MIC values have also showed low cytotoxicity. Moreover, two naphthoquinones derivatives 5 and 6 probably do not exhibit cross resistance with isoniazid and rifampicin, respectively, and regarding ADME analysis, no compound violated the Lipinski's rule-of-five. Considering the set of findings in this study, we conclude that these naphthoquinones could be promising scaffolds to develop new therapeutic strategies to TB.

Keywords: Mycobacterium tuberculosis, naphthoquinones, antimicrobials, resistance, tuberculosis

\section{INTRODUCTION}

According to World Health Organization, tuberculosis (TB) is one of the top 10 causes of death worldwide and, in 2016, there were an estimated the incidence of 10.4 million new cases (WHO, 2017). The main strategies for reducing TB morbidity and mortality rates are related to prophylaxis, early diagnosis and effective treatment of the disease. Bacille Calmette-Guérin (BCG) vaccine has been administered since the beginning of the 20th century in the immunization process, but only 
protects against the most severe cases of TB (Andersen and Woodworth, 2014; Stefanova, 2014). Despite the technological advances in the diagnostic area and the absence of an effective vaccine, the therapy still is the main tool for TB control.

The first-line therapy regimen for $\mathrm{TB}$ is a combination of four drugs: isoniazid (INH), rifampicin (RIF), pyrazinamide and ethambutol, which cure about $85 \%$ of patients infected with susceptible strains (WHO, 2017). Other antimicrobials such as kanamycin, amikacin, capreomycin, ethionamide, and levofloxacin are considered second-line drugs. These drugs are also used in the treatment of TB, especially in cases of multidrugresistant strains (MDR) infection - which are resistant to both INH and RIF - and when there is intolerance to first-line drugs. However, second-line therapy is more expensive, more toxic, and less effective than basic therapy (Ma et al., 2010).

Over the past 15 years, the TB treatment was able to avoid at least 50 million deaths, however, there is still a gap in this regard (WHO, 2017). Although it is a curable disease, there are several factors that hamper the TB control: lengthy treatment, toxic effects, pharmacokinetic interactions with other drugs, TB-HIV co-infection and the emergence of drug resistance (Palomino et al., 2009). Still in this context, the available therapeutic arsenal is insufficient, being ineffective against resistant strains and latent TB (Ma et al., 2010). Thus, new antimicrobial agents with novel mechanisms of action could be effective in the management of $\mathrm{TB}$, reducing cross-resistance, the length of treatment and the adverse effects. It would increase the patient compliancy to the therapy, favoring the cure (Hoagland et al., 2016; Igarashi et al., 2017).

The natural sources have been highlighted in the discovery and development of new antibiotics mainly due to structural diversity of the compounds, therapeutic potential and plethoric mechanisms of action (Bérdy, 2012; Newman and Cragg, 2016). Among the compounds that may be found in a variety of plants, algae, bacteria and fungi, are quinones: aromatic compounds which are classified in anthraquinones, benzoquinones and naphthoquinones, according to their chemical compositions (López-López et al., 2014). The naphthoquinones and derivatives have a naphthalene ring, and some studies have reported its antibacterial, antitumoral, antileishmanial, anthelmintic, and antifungal activity (Coelho et al., 2010; Hazra et al., 2013; Fiorito et al., 2014; Neto et al., 2014; Mata-Santos et al., 2015). Some mechanisms of action have been proposed for naphthoquinones, mainly related to oxidative stress - due to the production of reactive oxygen species (ROS) which induce apoptosis in biological systems (Da Silva et al., 2003) - and to inhibition of DNA gyrase (Karkare et al., 2013).

Previous research has demonstrated the antimycobacterial activity of naphthoquinones (Mital et al., 2008; Coelho et al., 2010; Ferreira et al., 2010; Cantos et al., 2012; Uc-Cachón et al., 2014), including against multidrug and extensively drug resistant strains (Dey et al., 2014), bringing attention to the potential of these compounds as a basis for the development of new anti-TB drugs. In this scenario, we aimed to evaluate the antimycobacterial activity of six 1,4-naphthoquinones derivatives against susceptible and drug resistant strains of Mycobacterium tuberculosis.

\section{MATERIALS AND METHODS}

\section{Synthesis}

The naphthoquinone $\mathbf{1}$ is commercial, and the other compounds were synthetized using methods previously described in the literature. Naphthoquinones $\mathbf{2}$ and $\mathbf{3}$ were synthesized via cyclization of lapachol and nor-lapachol, respectively (De Moura et al., 2001), while naphthoquinone 4 was synthesized via lawsone alilation in a two steps procedure (Pinto et al., 1997). The amination of 1,4-naphthoquinone with sodium azide resulted in the naphthoquinone 5 (Fieser and Hartwell, 1935) while the amination with aniline resulted in the naphthoquinone 6 (Lisboa et al., 2011) (Table 1). All compounds were solubilized in dimethyl sulfoxide $99.5 \%$ (Sigma-Aldrich) at a concentration of $10 \mathrm{mg} / \mathrm{ml}$ and stored at $4^{\circ} \mathrm{C}$ until used.

\section{Strains and Inoculum}

The antimicrobial activity of naphthoquinones was evaluated against three strains of $M$. tuberculosis: a pan-susceptible, $\mathrm{H} 37 \mathrm{Rv}$ (ATCC 27294); a mono-resistant to INH (INH ${ }_{\mathrm{R}}$ - ATCC 35822) with mutation in the kat $G$ gene (S315T); and a mono-resistant to RIF $\left(\mathrm{RMP}_{\mathrm{R}}\right.$ - ATCC 35838) with mutation in rpoB gene (H526Y). All strains were cultured in Ogawa-Kudoh, for up to 14 days at $37^{\circ} \mathrm{C}$ without $\mathrm{CO}_{2}$. The inoculum for each strain was prepared in a glass tube containing beads to break the

TABLE 1 | Characterization of the 1,4-naphthoquinone derivatives.

\begin{tabular}{|c|c|c|}
\hline Chemical structure & Chemical formula & Nomenclature \\
\hline 1 & $\mathrm{C}_{10} \mathrm{H}_{4} \mathrm{Cl}_{2} \mathrm{O}_{2}$ & $\begin{array}{l}\text { 2,3-Dichloronaphthalene- } \\
\text { 1,4-dione }\end{array}$ \\
\hline 2 & $\mathrm{C}_{15} \mathrm{H}_{14} \mathrm{O}_{3}$ & $\begin{array}{l}\text { 2,2-Dimethyl-3,4-dihydro- } \\
\text { 2H-benzo[g]chromene-5, } \\
\text { 10-dione }\end{array}$ \\
\hline 3 & $\mathrm{C}_{14} \mathrm{H}_{12} \mathrm{O}_{3}$ & $\begin{array}{l}\text { 2,2-Dimethyl-2,3- } \\
\text { dihydronaphtho[2,3-b]furan- } \\
\text { 4,9-dione }\end{array}$ \\
\hline 4 & $\mathrm{C}_{13} \mathrm{H}_{10} \mathrm{O}_{3}$ & $\begin{array}{l}\text { 2-(Allyloxy)naphthalene-1,4- } \\
\text { dione }\end{array}$ \\
\hline 5 & $\mathrm{C}_{10} \mathrm{H}_{7} \mathrm{NO}_{2}$ & $\begin{array}{l}\text { 2-Aminonaphthalene-1,4- } \\
\text { dione }\end{array}$ \\
\hline 6 & $\mathrm{C}_{16} \mathrm{H}_{11} \mathrm{NO}_{2}$ & $\begin{array}{l}\text { 2-(Phenylamino)naphthalene- } \\
\text { 1,4-dione }\end{array}$ \\
\hline
\end{tabular}


clumps, in sterile distilled water, according to $1.0 \mathrm{McF}$ arland scale $\left(3 \times 10^{8} \mathrm{UFC} / \mathrm{mL}\right)$ (Woods et al., 2011). After this process, it was diluted at a ratio of 1:20 in Middlebrook 7H9 Broth. The tests were performed at the Medical Microbiology Research Center (NUPEMM), at the Federal University of Rio Grande (FURG), under strict conditions required for handling M. tuberculosis.

\section{Resazurin Microtiter Assay (REMA)}

The minimum inhibitory concentration (MIC) of the compounds was determined by Resazurin Microtiter Assay (Palomino et al., 2002). Briefly, in a 96-well microplate, $100 \mu \mathrm{l}$ of $7 \mathrm{H} 9$ medium supplemented with 10\% OADC (Oleic acid Albumin Dextrose Catalase) was added in the test wells. In the first line was added $100 \mu \mathrm{l}$ of each compound previously diluted in sterile water; a serial microdilution (1:2) was performed in the $\mathrm{x}$-axis and, after, $100 \mu \mathrm{l}$ of the last well was discarded. At the end of microdilution was added $100 \mu \mathrm{L}$ of inoculum in each well and the final range of concentrations evaluated was 200 to $0.8 \mu \mathrm{g} / \mathrm{mL}$ for naphthoquinones, 8 to $0.03 \mu \mathrm{g} / \mathrm{mL}$ for $\mathrm{INH}$ and 1.024 to $0.06 \mu \mathrm{g} / \mathrm{mL}$ for RIF. In each plate were added sterility and growth controls. After incubation at $37^{\circ} \mathrm{C}$ for 7 days was added $30 \mu \mathrm{L}$ of resazurin $0.02 \%$ on the plate and then, $24 \mathrm{~h}$ later, the reading of the results was performed. MIC was defined as the lowest concentration of compound capable of inhibiting bacterial growth. All tests were performed in triplicate.

\section{Cytotoxicity Assay}

The cytotoxicity of the compounds was evaluated on adherent J774A.1 macrophage cell line (ATCC TIB-67). In a 96-well plate, $200 \mu \mathrm{L}$ of cell suspension (at the concentration of $1 \times 10^{5}$ cells/mL) were cultivated in Dulbecco's modified Eagle medium (Sigma-Aldrich) supplemented with $10 \%$ of fetal bovine serum and maintained for $24 \mathrm{~h}$ at $37^{\circ} \mathrm{C}$ in a humid atmosphere with $5 \% \mathrm{CO}_{2}$ (Snewin et al., 1999). After this period, the attached cells were exposed to different concentrations of naphthoquinones (200 to $0.8 \mu \mathrm{g} / \mathrm{mL}$ ) and the plate was again incubated overnight. To determinate the concentration of the compound capable of maintaining the viability of $50 \%$ of the cells $\left(\mathrm{IC}_{50}\right)$ were added $30 \mu \mathrm{L}$ of resazurin $0.01 \%$ and after $24 \mathrm{~h}$ of incubation the fluorescence was measured by Thermo Plate TP-Reader BioTek $^{\mathrm{TM}}$ ELx800 ${ }^{\mathrm{TM}}$ (Ansar Ahmed et al., 1994).

\section{Selectivity Index (SI)}

The SI of the compounds was calculated based on the results of MIC and $\mathrm{IC}_{50}$ of each compound for each strain, according to the following formula: $I C_{50} / M I C$. SI values equal to or greater than 10 indicate that the compound is pharmacologically safe (Orme, 2001; Pavan et al., 2012).

\section{Theoretical Properties of Absorption, Distribution, Metabolism, and Excretion (ADME)}

Characteristics of theoretical absorption, distribution, metabolism, and excretion (ADME) and toxicological effects of the compounds were determined by in silico analysis, using the free software: Molinspiration ${ }^{1}$, Swiss $\mathrm{ADME}^{2}$ (Daina et al., 2017), and OSIRIS Property Explorer ${ }^{3}$. According to the Lipinski Rule-of-Five, the following physicochemical parameters were evaluated: molecular weight, $\log \mathrm{P}, \mathrm{H}-$ bond donors, and $\mathrm{H}$-bond acceptors (Lipinski, 2004).

\section{Docking Analysis}

Flexible docking simulation was performed by ArgusLab 4.0.1, using Escherichia coli RNA polymerase as a protein template. The structures were from Protein Data Bank (PDB) ${ }^{4}$ - files 5UAC and 5UAQ. The interaction between proteins (wild-type and mutant H526Y) and the ligands (RMP and compound 6) was evaluated from residues 507 to 533, which comprise the RMP resistancedetermining region (RRDR) (Ramaswamy and Musser, 1998). In the docking calculations, it was applied the Ascore as scoring method (Luo et al., 2012).

\section{RESULTS}

All naphthoquinones showed inhibitory activity against the three M. tuberculosis strains with MIC ranging between 206.6 and $12.5 \mu \mathrm{M}$ (Table 2). Besides the naphthoquinones being active against the susceptible strain, the compounds also showed various levels of activity against the resistant strains (Table 2). The compounds $\mathbf{1}$ and $\mathbf{3}$ showed, respectively, MIC $=110.6$ and $54.8 \mu \mathrm{M}$, for all strains evaluated, while naphthoquinones 2 and 4 showed lower inhibitory concentrations against the susceptible strain, compared to the resistant strains. In addition, compounds 1, 2, and 4 exhibited $\mathrm{IC}_{50}$ between 103 and $285 \mu \mathrm{M}$, resulting in SI values between 0.07 and 2.8.

For the $\mathrm{INH}_{\mathrm{R}}$ strain, compared with the $\mathrm{H} 37 \mathrm{Rv}$, all compounds showed equal or reduced activity, except for compound 5. On the other hand, in comparison with the $\mathrm{RMP}_{\mathrm{R}}$ strain, the only difference in MIC was related to the compound 6 . Interestingly, the compound 6 was the most active against $\mathrm{RMP}_{\mathrm{R}}$ strain $(\mathrm{MIC}=12.5 \mu \mathrm{M})$, in a concentration eight times less than the concentration capable of inhibiting the growth of $\mathrm{H} 37 \mathrm{Rv}$ and $\mathrm{INH}_{\mathrm{R}}$ strains $(\mathrm{MIC}=110.4 \mu \mathrm{M})$.

The compound 6 was able to inhibit $\mathrm{RMP}_{\mathrm{R}}$ strain $(\mathrm{MIC}=12.5 \mu \mathrm{M})$ at a concentration $50 \mathrm{x}$ lower than the drug RMP $($ MIC $=622.2 \mathrm{uM})$ and, considering the peculiarity of this strain - which differs from the H37Rv mainly by the mutation in the rpoB gene (H526Y) - we were prompted to explore a possible affinity of this compound with mutant $r p o B$. By preliminary docking, we observed a reduction in the affinity between RMP and the RRDR site in mutant $r p o B$ target (Figure 1A) while compound 6 showed more negative free energy - strong binding (Silva et al., 2017) - compared to RMP for both wild-type as well as the mutant protein (Figure 1).

Regarding the ADME analysis, all the naphthoquinones evaluated in this study showed high gastrointestinal

\footnotetext{
${ }^{1}$ molinspiration.com/cgi-bin/properties

${ }^{2}$ http://www.swissadme.ch/index.php

${ }^{3}$ www.organic-chemistry.org/prog/peo/

${ }^{4}$ https://www.rcsb.org
} 
TABLE 2 | Activity of naphthoquinones against three M. tuberculosis strains and $\mathrm{IC}_{50}$ on $\mathrm{J} 774 \mathrm{~A} .1$ cells lineage.

\begin{tabular}{|c|c|c|c|c|c|c|c|}
\hline \multirow[t]{2}{*}{ Compound } & \multicolumn{3}{|c|}{ MIC ( $\mu \mathrm{M})$} & \multirow{2}{*}{$\frac{\mathrm{IC}_{50}(\mu \mathrm{M})}{\mathrm{J} 774 \mathrm{~A} .1}$} & \multicolumn{3}{|c|}{$\mathrm{SI}\left(I C_{50} / M I C\right)$} \\
\hline & H37Rv & $\mathrm{INH}_{\mathrm{R}}$ & $\mathbf{R M P}_{\mathbf{R}}$ & & H37Rv & $\mathrm{INH}_{\mathrm{R}}$ & $\mathrm{RMP}_{\mathrm{R}}$ \\
\hline 1 & 110.6 & 110.6 & 110.6 & 103 & 0.9 & 0.9 & 0.9 \\
\hline 2 & 103.3 & 206.6 & 206.6 & 285 & 2.8 & 1.4 & 1.4 \\
\hline 3 & 54.8 & 54.8 & 54.8 & $>877$ & $\geq 16$ & $\geq 16$ & $\geq 16$ \\
\hline 4 & 58.4 & 234 & 234 & 173 & 0.3 & 0.07 & 0.07 \\
\hline 5 & 72.2 & 36.1 & 36.1 & $>1156$ & $\geq 16$ & $\geq 32$ & $\geq 32$ \\
\hline 6 & 100.4 & 100.4 & 12.5 & $>803$ & $\geq 8$ & $\geq 8$ & $\geq 64$ \\
\hline $\mathrm{INH}$ & 0.438 & 14.6 & $\leq 0.219$ & 3733 & 8523 & 256 & ND \\
\hline $\mathrm{RIF}$ & 0.608 & 0.304 & 622.2 & $>2489$ & $\geq 4094$ & $\geq 8187$ & $\geq 4$ \\
\hline
\end{tabular}

MIC, minimum inhibitory concentration; IC50, inhibitory concentration of 50\%; SI, Selectivity Index; INHr, ATCC 35822; RMPr, ATCC 35838; ND, not determined.

A

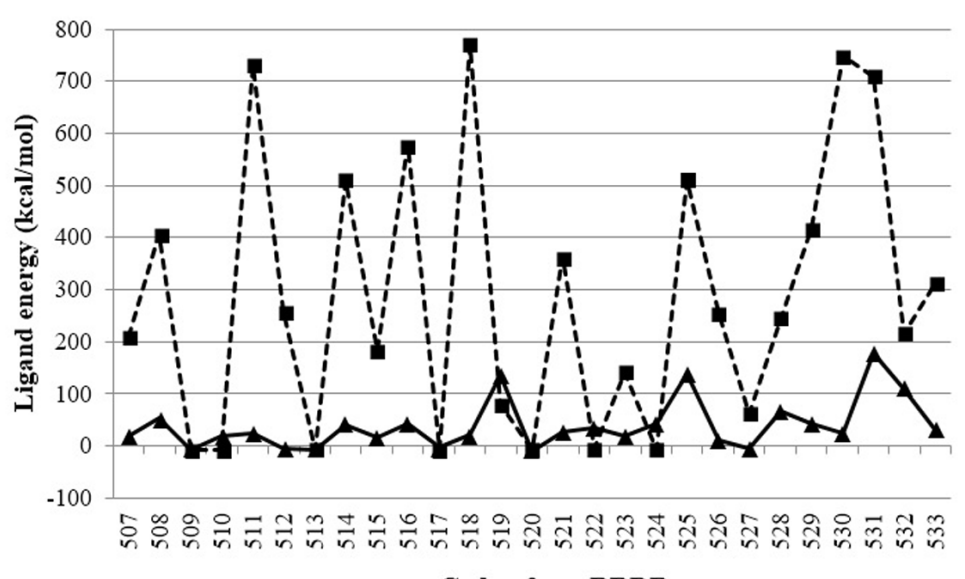

Codon from RRDR $\underset{\text { RMP }}{\operatorname{rpoB} W T+}$

--n-- rpoB H526Y +

RMP

\section{B}

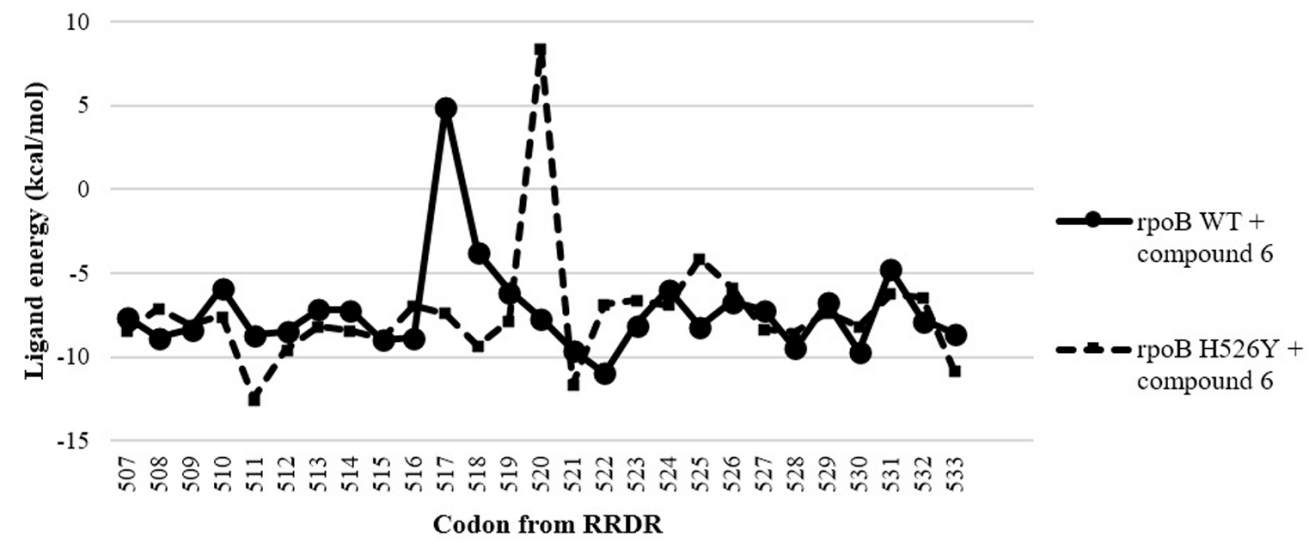

FIGURE 1 | Ligand energy between each codon from RRDR of Escherichia coli rpoB gene (WT and H526T) and RMP (A); and compound 6 (B).

absorption and are in agreement with the Lipinski's ruleof-five (Lipinski, 2004): molecular weight $\leq 500, \operatorname{miLogP} \leq 5$, H-bond donors $\leq 5$, and H-bond acceptors $\leq 10$ (Table 3), indicating crucial characteristics for oral bioavailability. In addition, most of the compounds showed none or low toxicity risk related to mutagenicity or tumorigenicity (Table 3).
In this study, by the pharmacokinetic analysis using the Software Swiss ADME, the theoretical inhibitory activity of naphthoquinone derivatives against five cytochrome P450 isoforms was evaluated (CYP450): CYP1A2, CYP2C9, CYP2C19, CYP2D6, and CYP3A4. All compounds showed potential inhibitory activity of CYP1A2 and CYP2C19 isoforms, except for compound 5, which showed interaction only with CYP1A2. 
TABLE 3 | Absorption, distribution, metabolism, and excretion (ADME) characterization and toxicity risks of naphthoquinones.

\begin{tabular}{|c|c|c|c|c|c|c|c|c|}
\hline Compound & MW & miLogP & TPSA $\left(\AA^{2}\right)$ & H-Acceptors & H-Donors & Gl absorption & Mutagenic & Tumorigenic \\
\hline 1 & 227.04 & 2.88 & 34.14 & 2 & 0 & High & Low & High \\
\hline 2 & 242.27 & 2.95 & 43.38 & 3 & 0 & High & None & None \\
\hline 3 & 228.24 & 2.43 & 43.38 & 3 & 0 & High & None & None \\
\hline 4 & 214.22 & 2.30 & 43.38 & 3 & 0 & High & Low & None \\
\hline 5 & 173.17 & 1.08 & 60.16 & 3 & 2 & High & High & None \\
\hline 6 & 249.26 & 3.59 & 46.17 & 3 & 1 & High & Low & None \\
\hline
\end{tabular}

MW, molecular weight; miLogP, octanol-water partition coefficient logP; TPSA, total polar surface area; Gl absorption, gastrointestinal absorption.

In addition, compounds $\mathbf{2}$ and $\mathbf{6}$ also showed activity against the $3 \mathrm{~A} 4$ isoform.

\section{DISCUSSION}

We evaluated the antimycobacterial potential of six 1,4naphthoquinone derivatives: all compounds were active against susceptible and resistant strains of $M$. tuberculosis, with different MIC values. Mathew et al. (2010) reported the antimycobacterial activity of plumbagin and its derivatives, and Dey et al. (2014) reported the susceptibility of mycobacteria (sensitive and resistant) to naphthoquinones, mainly plumbagin, with MIC between 0.25 and $16 \mu \mathrm{g} / \mathrm{mL}$. Thus, both highlight the potential of naphthoquinones in the search for new antiTB drugs. Our research group has developed several studies with a focus on new antimicrobial agents and, although other studies have reported the activity of other naphthoquinone derivatives, including for resistant strains, their mechanism of action is still uncertain (Coelho et al., 2010; Jardim et al., 2015).

Based on the analysis of the structure-activity relationship, the compound 3 , which has a tetrahydrofuran radical and anti-trypanosome activity previously described (De Moura et al., 2001), showed MIC $=54.8 \mu \mathrm{M}$ against the three M. tuberculosis strains, while the compound 2, which contains a tetrahydropyran radical, showed MIC between 103.3 and $206.6 \mu \mathrm{M}$. Besides showing a better antimycobacterial activity, the compound 3 has also shown reduced cytotoxicity $\left(\mathrm{IC}_{50}>877 \mu \mathrm{M}\right)$ compared with $2\left(\mathrm{IC}_{50}=285 \mu \mathrm{M}\right)$, and both showed none mutagenic or tumorigenic risks (Table 3).

When we analyzed the compounds with nitrogen (5 and 6), it was noticed that phenylamine radical in the compound 6 has decreased the activity for the susceptible and $\mathrm{INH}_{\mathrm{R}}$ strains, while was able to a threefold increase the activity of this naphthoquinone for $\mathrm{RMP}_{\mathrm{R}}$ strain, compared with compound 5, which has the amine group (Table 2 ). The activity of naphthoquinones with nitrogenous radicals also has been described against fungi, gram positive and negative bacteria (Riffel et al., 2002; Rahmoun et al., 2013).

Among the rifampicin-resistant $M$. tuberculosis, about 35\% have the mutation H526Y (Molodtsov et al., 2017) and, considering that compound 6 was active against the $\mathrm{RMP}_{\mathrm{R}}$ strain, we can infer that there is probably no cross-resistance between this compound and RMP, what was also evidenced in the docking analysis (Molodtsov et al., 2017). Moreover, due to the lower binding energies of the compound $\mathbf{6}$ when compared to the binding energies of RIF in RRDR, the mutation seems to favor the binding between the naphthoquinone 6 and the RRDR (Figure 1).

Furthermore, among the naphthoquinone derivatives evaluated, the compound 5 was the most active for the $\mathrm{INH}_{\mathrm{R}}$ strain $(\mathrm{MIC}=36.1 \mu \mathrm{M})$, which has a mutation in the kat $G$ gene (S315T). This result evidences the absence of cross-resistance of this compound with INH, especially in strains that have this mutation.

According to the results of in silico pharmacokinetics, the naphthoquinone derivatives have inhibitory potential in some human CYP450 isoforms. It is known that the electron transfer chain is directly related with activity of enzymes, such as $\mathrm{NADH}$, transferring electrons from lipophilic redox carriers. In this context, the importance of this biosynthetic route as a target in the search for new antimicrobials has been highlighted (Black et al., 2014) and, considering the results obtained in this study, the naphthoquinones could act as other quinones previously described, in the transfer chain of electrons (Heikal et al., 2016).

\section{CONCLUSION}

Considering the potential of quinones as a source of new antimicrobial agents and their biological activities already reported, this set of findings confirm this hypothesis and demonstrate that, naphthoquinones derivatives could be scaffolds for the development of new anti-TB drugs.

\section{AUTHOR CONTRIBUTIONS}

PH, DR, and PdS designed the study. KDM, PC, KDR, TC, and MP contributed with compounds synthesis. PH, LF, and DR performed the experiments. $\mathrm{PH}, \mathrm{DR}, \mathrm{PdS}$, and KDM analyzed the data and wrote the manuscript. All authors approved the version to be published.

\section{ACKNOWLEDGMENTS}

This work was supported by National Council for Technological and Scientific Development $(\mathrm{CNPq})$ and Coordination for the Improvement of Higher Education Personnel (CAPES). 


\section{REFERENCES}

Andersen, P., and Woodworth, J. S. (2014). Tuberculosis vaccines - rethinking the current paradigm. Trends Immunol. 35, 387-395. doi: 10.1016/j.it.2014.04.006

Ansar Ahmed, S., Gogal, R. M., and Walsh, J. E. (1994). A new rapid and simple non-radioactive assay to monitor and determine the proliferation of lymphocytes: an alternative to [3H]thymidine incorporation assay. J. Immunol. Methods 170, 211-224. doi: 10.1016/0022-1759(94)90396-4

Bérdy, J. (2012). Thoughts and facts about antibiotics: where we are now and where we are heading. J. Antibiot. 65, 441-441. doi: 10.1038/ja.2012.54

Black, P. A., Warren, R. M., Louw, G. E., Van Helden, P. D., Victor, T. C., and Kana, B. D. (2014). Energy metabolism and drug efflux in Mycobacterium tuberculosis. Antimicrob. Agents Chemother. 58, 2491-2503. doi: 10.1128/AAC.02293-13

Cantos, J. B., Coelho, T. S., Carneiro, P. F., de Moura, K. C. G., Pinto, M. C. F. R., da Silva Júnior, E. N., et al. (2012). Antimicrobial evaluation of quinones and heterocyclic compounds against Mycobacterium marinum, M. kansasii and M. abscessus. Lat. Am. J. Pharm. 31, 507-513.

Coelho, T. S., Silva, R. S. F., Pinto, A. V., Pinto, M. C. F. R., Scaini, C. J., Moura, K. C. G., et al. (2010). Activity of $\beta$-lapachone derivatives against rifampicinsusceptible and -resistant strains of Mycobacterium tuberculosis. Tuberculosis 90, 293-297. doi: 10.1016/j.tube.2010.06.001

Da Silva, M. N., Ferreira, V. F., and De Souza, M. C. B. V. (2003). Um panorama atual da química e da farmacologia de naftoquinonas, com ênfase na $\beta$-Lapachona e derivados. Quim. Nova 26, 407-416. doi: 10.1590/S010040422003000300019

Daina, A., Michielin, O., and Zoete, V. (2017). SwissADME: a free web tool to evaluate pharmacokinetics, drug-likeness and medicinal chemistry friendliness of small molecules. Sci. Rep. 7:42717. doi: 10.1038/srep42717

De Moura, K. C. G., Emery, F. S., Neves-Pinto, C., Pinto, M. D. C. F. R., Dantas, A. P., Salomão, K., et al. (2001). Trypanocidal activity of isolated naphthoquinones from Tabebuia and some heterocyclic derivatives: a review from an interdisciplinary study. J. Braz. Chem. Soc. 12, 325-338. doi: 10.1590/ S0103-50532001000300003

Dey, D., Ray, R., and Hazra, B. (2014). Antitubercular and antibacterial activity of quinonoid natural products against multi-drug resistant clinical isolates. Phyther. Res. 28, 1014-1021. doi: 10.1002/ptr.5090

Ferreira, S. B., de Carvalho da Silva, F., Bezerra, F. A. F. M., Lourenço, M. C. S., Kaiser, C. R., Pinto, A. C., et al. (2010). Synthesis of alpha- and beta-pyran naphthoquinones as a new class of antitubercular agents. Arch. Pharm. 343, 81-90. doi: 10.1002/ardp. 200900162

Fieser, L. F., and Hartwell, J. L. (1935). The reaction of hydrazoic acid with the naphthoquinones. J. Am. Chem. Soc. 57, 1482-1484. doi: 10.1021/ja01311a030

Fiorito, S., Epifano, F., Bruyère, C., Mathieu, V., Kiss, R., and Genovese, S. (2014). Growth inhibitory activity for cancer cell lines of lapachol and its natural and semi-synthetic derivatives. Bioorg. Med. Chem. Lett. 24, 454-457. doi: 10.1016/ j.bmcl.2013.12.049

Hazra, S., Ghosh, S., Das Sarma, M., Sharma, S., Das, M., Saudagar, P., et al. (2013). Evaluation of a diospyrin derivative as antileishmanial agent and potential modulator of ornithine decarboxylase of Leishmania donovani. Exp. Parasitol. 135, 407-413. doi: 10.1016/j.exppara.2013.07.021

Heikal, A., Hards, K., Cheung, C. Y., Menorca, A., Timmer, M. S. M., Stocker, B. L., et al. (2016). Activation of type II NADH dehydrogenase by quinolinequinones mediates antitubercular cell death. J. Antimicrob. Chemother. 71, 2840-2847. doi: $10.1093 / \mathrm{jac} / \mathrm{dkw} 244$

Hoagland, D. T., Liu, J., Lee, R. B., and Lee, R. E. (2016). New agents for the treatment of drug-resistant Mycobacterium tuberculosis. Adv. Drug Deliv. Rev. 102, 55-72. doi: 10.1016/j.addr.2016.04.026

Igarashi, M., Ishizaki, Y., and Takahashi, Y. (2017). New antituberculous drugs derived from natural products: current perspectives and issues in antituberculous drug development. J. Antibiot. doi: 10.1038/ja.2017.126 [Epub ahead of print].

Jardim, G. A. M., Cruz, E. H. G., Valença, W. O., Resende, J. M., Rodrigues, B. L., Ramos, D. F., et al. (2015). On the search for potential antimycobacterial drugs: synthesis of naphthoquinoidal, phenazinic and 1,2,3-triazolic compounds and evaluation against Mycobacterium tuberculosis. J. Braz. Chem. Soc. 26, 1013-1027. doi: 10.5935/0103-5053.20150067

Karkare, S., Chung, T. T. H., Collin, F., Mitchenall, L. A., McKay, A. R., Greive, S. J., et al. (2013). The naphthoquinone diospyrin is an inhibitor of DNA gyrase with a novel mechanism of action. J. Biol. Chem. 288, 5149-5156. doi: 10.1074/jbc.M112.419069

Lipinski, C. A. (2004). Lead- and drug-like compounds: the rule-of-five revolution. Drug Discov. Today Technol. 1, 337-341. doi: 10.1016/j.ddtec.2004.11.007

Lisboa, C. D. S., Santos, V. G., Vaz, B. G., De Lucas, N. C., Eberlin, M. N., and Garden, S. J. (2011). C-H functionalization of 1,4-naphthoquinone by oxidative coupling with anilines in the presence of a catalytic quantity of copper(II) acetate. J. Org. Chem. 76, 5264-5273. doi: 10.1021/jo200354u

López-López, L. I., Nery-Flores, D. S., Silva-Belmares, Y. S., and Sáenz-Galindo, A. (2014). Naphthoquinones: biological properties and synthesis of lawsone and derivatives - A structured review. Vitae 21, 248-258.

Luo, H. J., Wang, J. Z., Zhou, Y., and Zou, K. (2012). Docking study on trametenolic acid B as a $\alpha$-glucosidase inhibitor. Med. Chem. Res. 21, 2141-2144. doi: 10.1007/s00044-011-9741-y

Ma, Z., Lienhardt, C., McIlleron, H., Nunn, A. J., and Wang, X. (2010). Global tuberculosis drug development pipeline: the need and the reality. Lancet 375 , 2100-2109. doi: 10.1016/S0140-6736(10)60359-9

Mata-Santos, T., Pinto, N. F., Mata-Santos, H. A., De Moura, K. G., Carneiro, P. F., Carvalho, T. D. S., et al. (2015). Anthelmintic activity of lapachol, $\beta$-lapachone and its derivatives against Toxocara canis larvae. Rev. Inst. Med. Trop. Sao Paulo 57, 197-204. doi: 10.1590/S0036-46652015000300003

Mathew, R., Kruthiventi, A. K., Prasad, J. V., Kumar, S. P., Srinu, G., and Chatterji, D. (2010). Inhibition of mycobacterial growth by plumbagin derivatives. Chem. Biol. Drug Des. 76, 34-42. doi: 10.1111/j.1747-0285.2010. 00987.x

Mital, A., Negi, V. S., and Ramachandran, U. (2008). Synthesis and biological evaluation of substituted naphthoquinone derivatives as potent antimycobacterial agents. ARKIVOC 2008, 176-192. doi: 10.3998/ark. 5550190.0009.f17

Molodtsov, V., Scharf, N. T., Stefan, M. A., Garcia, G. A., and Murakami, K. S. (2017). Structural basis for rifamycin resistance of bacterial RNA polymerase by the three most clinically important RpoB mutations found in Mycobacterium tuberculosis. Mol. Microbiol. 103, 1034-1045. doi: 10.1111/mmi.13606

Neto, J. B. A., Da Silva, C. R., Neta, M. A. S., Campos, R. S., Siebra, J. T., Silva, R. A. C., et al. (2014). Antifungal activity of naphthoquinoidal compounds in vitro against fluconazole-resistant strains of different Candida species: a special emphasis on mechanisms of action on Candida tropicalis. PLoS One 9:e93698. doi: 10.1371/journal.pone.0093698

Newman, D. J., and Cragg, G. M. (2016). Natural products as sources of new drugs from 1981 to 2014. J. Nat. Prod. 79, 629-661. doi: 10.1021/acs.jnatprod.5b01055

Orme, I. (2001). Search for new drugs for treatment of tuberculosis. Antimicrob. Agents Chemother. 45, 1943-1946. doi: 10.1128/AAC.45.7.1943-1946.2001

Palomino, J.-C., Martin, A., Camacho, M., Guerra, H., Swings, J., and Portaels, F. (2002). Resazurin microtiter assay plate: simple and inexpensive method for detection of drug resistance in Mycobacterium tuberculosis. Antimicrob. Agents Chemother. 46, 2720-2722. doi: 10.1128/AAC.46.8.2720-2722.2002

Palomino, J. C., Ramos, D. F., Almeida, P., and Silva, D. (2009). New antituberculosis drugs: strategies, sources and new molecules. Curr. Med. Chem. 16, 1898-1904. doi: 10.2174/092986709788186066

Pavan, F. R., Sato, D. N., and Leite, C. Q. F. (2012). "An approach to the search for new drugs against tuberculosis," in Understanding Tuberculosis - New Approaches to Fighting against Drug Resistance, ed. P.-J. Cardona (London: IntechOpen), 137-146.

Pinto, A. V., Pinto, C. N., Pinto Mdo, C., Rita, R. S., Pezzella, C. A., et al. (1997). Trypanocidal activity of synthetic heterocyclic derivatives of active quinones from Tabebuia sp. Arzneimittelforschung 47, 74-79.

Rahmoun, N. M., Boucherit-Atmani, Z., Benabdallah, M., Boucherit, K., Villemin, D., and Choukchou-Braham, N. (2013). Antimicrobial activities of the henna extract and some synthetic naphthoquinones derivatives. Am. J. Med. Biol. Res. 1, 16-22. doi: 10.12691/ajmbr-1-1-3

Ramaswamy, S., and Musser, J. M. (1998). Molecular genetic basis of antimicrobial agent resistance in Mycobacterium tuberculosis: 1998 update. Tuber. Lung Dis. 79, 3-29. doi: 10.1054/tuld.1998.0002

Riffel, A., Medina, L. F., Stefani, V., Santos, R. C., Bizani, D., and Brandelli, A. (2002). In vitro antimicrobial activity of a new series of 1,4-naphthoquinones. Braz. J. Med. Biol. Res. 35, 811-818. doi: 10.1590/S0100-879X2002000700008

Silva, L., Carrion, L. L., von Groll, A., Costa, S. S., Junqueira, E., Ramos, D. F., et al. (2017). In vitro and in silico analysis of the efficiency 
of tetrahydropyridines as drug efflux inhibitors in Escherichia coli. Int. J. Antimicrob. Agents 49, 308-314. doi: 10.1016/j.ijantimicag.2016. 11.024

Snewin, V. A., Gares, M. P., Gaora, P. Ó., Hasan, Z., Brown, I. N., and Young, D. B. (1999). Assessment of immunity to mycobacterial infection with luciferase reporter constructs. Infect. Immun. 67, 4586-4593.

Stefanova, T. (2014). Quality control and safety assessment of BCG vaccines in the post-genomic era. Biotechnol. Biotechnol. Equip. 28, 387-391. doi: 10.1080/ 13102818.2014.927200

Uc-Cachón, A. H., Borges-Argáez, R., Said-Fernández, S., Vargas-Villarreal, J., González-Salazar, F., Méndez-González, M., et al. (2014). Naphthoquinones isolated from Diospyros anisandra exhibit potent activity against pan-resistant first-line drugs Mycobacterium tuberculosis strains. Pulm. Pharmacol. Ther. 27, 114-120. doi: 10.1016/j.pupt.2013.08.001

WHO (2017). WHO Global Tuberculosis Report 2017. Geneva: World Health Organization.
Woods, G. L., Brown-Elliott, B. A., Conville, P. S., Desmond, E. P., Hall, G. S., and Lin, G. (2011). Susceptibility testing of mycobacteria, nocardiae, and other aerobic actinomycetes; approved standard-second edition. Clin. Lab. Stand. Inst. 26, 1-61. doi: 10.1306/E4FD4657-1732-11D7-8645000102C1865D

Conflict of Interest Statement: The authors declare that the research was conducted in the absence of any commercial or financial relationships that could be construed as a potential conflict of interest.

Copyright $\odot 2018$ Halicki, Ferreira, De Moura, Carneiro, Del Rio, Carvalho, Pinto, da Silva and Ramos. This is an open-access article distributed under the terms of the Creative Commons Attribution License (CC BY). The use, distribution or reproduction in other forums is permitted, provided the original author(s) and the copyright owner are credited and that the original publication in this journal is cited, in accordance with accepted academic practice. No use, distribution or reproduction is permitted which does not comply with these terms. 